\title{
Objectively Quantified Physical Activity and Sedentary Behavior in Predicting Visceral Adiposity and Liver Fat
}

\author{
Shelley E. Keating, ${ }^{1,2}$ Helen M. Parker, ${ }^{1}$ Toby G. Pavey, ${ }^{2}$ Michael K. Baker, ${ }^{3}$ \\ Ian D. Caterson, ${ }^{4}$ Jacob George, ${ }^{5}$ and Nathan A. Johnson ${ }^{1,4}$ \\ ${ }^{1}$ Faculty of Health Sciences, University of Sydney, Lidcombe, NSW, Australia \\ ${ }^{2}$ School of Human Movement and Nutrition Sciences, The University of Queensland, Brisbane, QLD, Australia \\ ${ }^{3}$ School of Exercise Science, Australian Catholic University, Sydney, NSW, Australia \\ ${ }^{4}$ Boden Institute of Obesity, Nutrition, Exercise and Eating Disorders, University of Sydney, Sydney, NSW, Australia \\ ${ }^{5}$ Storr Liver Centre, Westmead Millennium Institute and Westmead Hospital, University of Sydney, Westmead, NSW, Australia
}

Correspondence should be addressed to Nathan A. Johnson; nathan.johnson@sydney.edu.au

Received 2 April 2016; Revised 5 August 2016; Accepted 25 August 2016

Academic Editor: Eliot Brinton

Copyright (C) 2016 Shelley E. Keating et al. This is an open access article distributed under the Creative Commons Attribution License, which permits unrestricted use, distribution, and reproduction in any medium, provided the original work is properly cited.

\begin{abstract}
Objective. Epidemiologic studies suggest an inverse relationship between nonalcoholic fatty liver disease (NAFLD), visceral adipose tissue (VAT), and self-reported physical activity levels. However, subjective measurements can be inaccurate and prone to reporter bias. We investigated whether objectively quantified physical activity levels predicted liver fat and VAT in overweight/obese adults. Methods. Habitual physical activity was measured by triaxial accelerometry for four days $(n=82)$. Time spent in sedentary behavior $($ MET $<1.6)$ and light (MET $1.6<3$ ), moderate (MET $3<6$ ), and vigorous (MET $6<9$ ) physical activity was quantified. Magnetic resonance imaging and spectroscopy were used to quantify visceral and liver fat. Bivariate correlations and hierarchical multiple regression analyses were performed. Results. There were no associations between physical activity or sedentary behavior and liver lipid. Sedentary behavior and moderate and vigorous physical activity accounted for just 3\% of variance for VAT $(p=0.14)$ and $0.003 \%$ for liver fat $(p=0.96)$. Higher levels of VAT were associated with time spent in moderate activity $(r=0.294, p=0.007)$, but there was no association with sedentary behavior. Known risk factors for obesity-related NAFLD accounted for $62 \%$ and $40 \%$ of variance in VAT and liver fat, respectively $(p<0.01)$. Conclusion. Objectively measured levels of habitual physical activity and sedentary behavior did not influence VAT or liver fat.
\end{abstract}

\section{Introduction}

The benefits of regular structured exercise are well documented and include improvements in cardiovascular and metabolic function, which reduce the risk of chronic health conditions such as type 2 diabetes and cardiovascular disease [1]. Recent evidence also demonstrates that exercise alone (in the absence of concomitant dietary restriction) reduces visceral adipose tissue (VAT) [2] and intrahepatic lipid (IHL) [3-5], both of which are independently associated with cardiovascular and metabolic risk [6]. While there is strong evidence for the benefits of regular structured exercise (i.e., exercise which is planned and involves repetitive movement), it is currently unclear whether increased levels of habitual activity (activity that is unstructured and accumulated during daily activities outside of structured exercise) and reduced sedentary behavior (activity with low metabolic rate $(<1.6$ METS) and in a reclining or seated position) have similar health benefits in the reduction of these adipose tissue depots. Understanding such associations has implications for policy as, because of low participation rates in structured exercise [7], there is an increasing emphasis in public health messages on the importance of habitual physical activity levels and reduced sedentary time [8].

Cross-sectional analyses indicate an inverse relationship between central adiposity as inferred by waist circumference [9], dual energy X-ray absorptiometry [10], or computed tomography (CT) [11] and self-reported physical activity 
levels. A similar inverse relationship has been observed for nonalcoholic fatty liver disease (NAFLD) [12-14]. Prospective studies have further supported this association [15-17]. In the Finnish Twin Cohort (TWINACTIVE) study, physically inactive cotwins were found to have liver fat scores (assessed via difference in mean signal intensity from magnetic resonance imaging) $170 \%$ greater than that of their active cotwin [17], and habitual physical activity, performed outside of work hours, was the strongest correlate of central abdominal fat after controlling for both environmental and genetic factors [18]. However, self-reported behavior is subject to reporter bias and tends to overestimate activity levels [19], especially in overweight and obese populations [20]. Emerging evidence using objectively measured physical activity via accelerometry supports these observations [21-24]; however research using accurate assessments of VAT and IHL is limited.

If the above epidemiological data [11-14] can be confirmed by high quality objective assessments, encouraging habitual physical activity and reducing sedentary behavior will have important clinical ramifications. Hence, in this study, we aimed to evaluate whether levels of accurately quantified sedentary behavior and habitual physical activity could predict levels of VAT and IHL in adults with overweight/obesity. Based on current guidelines for the management of obesity which recommend high volumes of moderate intensity activity [25], we hypothesised that there would be a negative relationship between time spent in moderate intensity physical activity and levels of VAT and IHL and a positive relationship between time spent in sedentary behavior and VAT and IHL content. Furthermore, we hypothesised that levels of sedentary behavior and moderate physical activity would explain a significant proportion of variance in VAT and IHL when modelled with known obesity-related health risk factors (including age, sex, body mass index (BMI), waist circumference, fasting glucose, fasting insulin, high density lipoprotein cholesterol, triglycerides, and resting blood pressure).

\section{Methods}

2.1. Participants. Participants were recruited via noticeboards, electronic bulletins, and clinical databases between June 2011 and January 2015 from a community-based sample of adult men and women (aged 18-60 years) with overweight or obesity (BMI $\geq 25.0 \mathrm{~kg} \cdot \mathrm{m}^{-2}$ ). Volunteers were excluded if they reported being physically active (exercising $>3$ days per week or meeting current guidelines for physical activity [1]) or were cigarette smokers. Participants provided clearance for participation from a medical practitioner prior to involvement and were excluded if there was evidence of an unstable cardiac condition, type 2 diabetes, hypertension, or liver disease other than nonalcoholic fatty liver disease (NAFLD) or secondary causes of steatohepatitis. Other exclusion criteria included taking lipid-lowering or insulin sensitizing medication, reporting taking $n-3$ polyunsaturated fatty acids within the last 6 months, or a change in medication within the last 12 months, regular alcohol consumption $>20 \mathrm{~g} /$ day, or a significant change in body weight
( $>5 \%$ weight change) in the previous three months. The analysis included 82 volunteers from larger intervention trials (ANZCTRN12610000351011; ACTRN12614000723684), the results of which have been published elsewhere [5]. The study conformed to the ethical guidelines of the $1975 \mathrm{Dec}-$ laration of Helsinki and was approved by The University of Sydney Human Research Ethics Committee and the Sydney Local Health District Ethics Review Committee. Eligible participants were screened via telephone interview and those meeting criteria provided informed consent and were invited to attend baseline assessments which included measures of anthropometry, a fasting blood test, magnetic resonance imaging (MRI) and spectroscopy $\left({ }^{1} \mathrm{H}-\mathrm{MRS}\right)$, and accelerometry.

\subsection{Measurements}

2.2.1. Anthropometry and Blood Pressure Assessment. Stature was recorded by stadiometer (SECA model 220 Telescopic Height Rod, Hamburg, Germany). Weight was measured (Tanita BC-418 Body Composition Analyzer; Tanita Corporation, Tokyo, Japan) to the nearest $0.1 \mathrm{~kg}$ and BMI $\left(\mathrm{kg} \cdot \mathrm{m}^{-2}\right)$ was calculated. Waist circumference was measured in triplicate at the horizontal plane, midway between the inferior margin of the ribs and the superior boarder of the iliac crest at end-expiration. Systolic (SBP) and diastolic (DBP) blood pressure were measured manually on each arm, after 10$15 \mathrm{~min}$ of quiet sitting.

2.2.2. Biochemical Parameters. Venous blood was collected from the antecubital vein after an overnight fast ( $>10$ hours). On the same day serum glucose, insulin, total cholesterol (TC), triglycerides (TG), high density lipoprotein cholesterol (HDL), low density lipoprotein cholesterol (LDL), alanine aminotransferase (ALT), aspartate aminotransferase (AST), and high sensitivity C-reactive protein (hs-CRP) were analysed by a commercially accredited laboratory.

2.2.3. Magnetic Resonance Imaging (MRI) and Proton Magnetic Resonance Spectroscopy $\left({ }^{1} H\right.$-MRS). Visceral adipose tissue was assessed via MRI and intrahepatic lipid via ${ }^{1} \mathrm{H}$-MRS using a 1.5 Tesla Achieva whole-body system (Philips Medical Systems; Best, Netherlands) as previously outlined $[5,26]$. The cross-sectional areas for abdominal VAT were analysed using automated software (Hippo $\mathrm{Fat}^{\mathrm{TM}}$ ) [27] with manual editing as necessary. Total VAT volumes were calculated by summation of slice areas with adjustment for slice thickness. Liver spectra data were analysed by magnetic resonance user interface software (jMRUI version 4.0, EU Project) [26]. Hepatic water signal amplitude was measured from the nonwater suppressed spectrum [5].

2.2.4. Objective Activity Monitoring. Current mean time spent in physical activity, sedentary behavior, daily steps, and energy expenditure were assessed by triaxial accelerometer (SenseWear ${ }^{\mathrm{TM}}$, BodyMedia Inc., PA, USA). Data were collected from all participants for four days (3 weekdays and 1 weekend, which was the minimum inclusion criteria) for 24 hours per day, except during water-based activities 
including showering, bathing, or swimming. All data were collected at least 7 days after magnetic resonance imaging. Armbands were worn on the left upper arm. Participants were instructed to continue with their habitual routine during this period. Physical activity levels and sedentary behavior were quantified (BodyMedia software, v8.0) with sedentary behavior defined as <1.6 METs, "light" activity as $1.6<$ 3 METs, "moderate" activity as $3<6$ METs, "vigorous" activity as $6<9$ METs, and "high" activity as $\geq 9$ METs [28]. Sleep duration was not included in time spent in sedentary behavior. Data were included if the monitor was worn for $>85 \%$ of a 24 -hour day.

2.3. Statistical Analysis. All descriptive data are reported as means \pm standard error from the mean (SE). Differences between groups were compared by Student's $t$-test with homogeneity of variances assessed via Levene's test for equality of variance or $\chi^{2}$ for categorical data. Pearson coefficients $(R)$ were used for all correlations. Separate hierarchical multiple regression analyses were performed for prediction of VAT and IHL percentage. Simple anthropometric and demographic variables were entered at Block 1 for both VAT and IHL. Known cardiometabolic risk variables were entered into the regression at Block 2, based on the variables that were significantly associated with VAT and IHL, respectively, in our sample (Table 2). These included TG, glucose, insulin, SBP, DBP, ALT and BMI for VAT and glucose, insulin, ALT, HDL, and BMI for IHL. While TG was not significantly associated with VAT in our analysis, it was included given its previously established association with excess VAT [29]. Finally, mean time spent in sedentary behavior, moderate intensity activity, and vigorous intensity activity were entered into Block 3 of the regression analysis. Data were analysed using Statistical Package for the Social Sciences (SPSS version 22.0; IMB Corp., Armonk, NY, USA); $p$ values were based on two-sided tests and considered statistically significant at $p<0.05$.

\section{Results}

3.1. Participants. Eighty-two adults ( $60 \%$ male) participated in the study. Mean age was $40.1 \pm 1.9$ years, BMI $30.9 \pm$ $0.5 \mathrm{~kg} \cdot \mathrm{m}^{-2}$, waist circumference $100.0 \pm 1.1 \mathrm{~cm}$, and liver fat $5.3 \pm 0.5 \%$. Thirty-five percent of the participants were classified as having NAFLD based on IHL > 5.5\% [30]. Other participants' characteristics are summarised in Table 1. Mean device wear time was $97 \pm 2 \%$ of a 24 -hour day. Participants with NAFLD had a significantly higher BMI, waist circumference, SBP, VAT, fasting glucose, and fasting insulin $(p<0.05)$. There were no differences in objectively measured levels of physical activity, sedentary behavior, total daily energy expenditure, or the number of steps taken per day between participants with and without NAFLD (Table 1). Time spent in high intensity activity ( $>9$ METS) was negligible (mean $37 \pm 27$ seconds) with only $3 / 82$ participants (4\%) engaging in high intensity activity for more than an average of one minute in total. When high intensity activity was included in the analysis, the results were not altered (data not shown). While there are no established critical thresholds for VAT volume with respect to morbidity and mortality risk, based on gender specific waist circumferences for Caucasian populations, $51 \%$ of men and $73 \%$ of women were above the risk threshold for abdominal obesity [31].

3.2. Bivariate Correlations. Bivariate correlations between VAT and IHL and biochemical, anthropometric, and accelerometry assessed activity variables are summarised in Table 2. A higher volume of VAT was associated with higher IHL, BMI, waist circumference, SBP, DBP, fasting glucose, fasting insulin, and ALT (Table 2). There was a weak association between higher levels of VAT and higher amounts of moderate intensity physical activity $(r=0.294$, $p=0.007)$. Higher levels of IHL were associated with higher BMI, waist circumference, VAT, fasting glucose, and fasting insulin and lower levels of HDL, but there was no association between IHL and total energy expended, time spent in sedentary behavior, and time spent in any level of activity (Table 2).

\subsection{Hierarchical Multiple Regression Analysis. Demographic} and anthropometric variables (waist circumference, sex, and age) accounted for $54 \%$ and $30 \%$ of the variance in VAT and IHL, respectively ( $p<0.001$; Tables 3 and 4$)$. The addition of TG, glucose, insulin, SBP, DBP, and BMI in Block 2 accounted for a further $9 \%$ of variance in VAT $(p=0.032)$. The addition of glucose, HDL, ALT, insulin, and BMI increased the prediction of IHL to $40 \%$; however this was not significant $(p=0.06)$. The addition of time spent in sedentary behavior, moderate and vigorous intensity activity (Block 3), increased the prediction of VAT to $65 \%$ $(p=0.14)$ and did not change the prediction of IHL $(p=0.96)$. Waist circumference, triglyceride, and fasting glucose contributed significantly to the prediction of high levels of VAT while waist circumference and fasting insulin contributed significantly to the prediction of high levels of IHL.

\section{Discussion}

We aimed to evaluate whether levels of accurately quantified sedentary behavior and habitual physical activity could predict levels of visceral adiposity and liver fat. Surprisingly, while traditional risk factors for cardiovascular disease (e.g., sex, age, and waist circumference) were associated with VAT and IHL, no relationship was observed between objectively measured physical inactivity and raised liver fat. Further, we unexpectedly observed a weak positive association between VAT volume and time spent in moderate activity (i.e., increased VAT was associated with higher time spent in moderate intensity physical activity) and also total energy expenditure. The inclusion of objectively measured levels of moderate activity and sedentary behavior did not improve the potency of traditional risk factors (waist circumference, triglycerides, fasting glucose, or fasting insulin) for predicting the liver fat concentration or level of visceral adiposity. As a cross-sectional analysis, this study demonstrates that current 
TABLE 1: Participants' characteristics.

\begin{tabular}{|c|c|c|c|c|}
\hline & Total group $(n=82)$ & $\operatorname{NAFLD}(n=29)$ & Non-NAFLD $(n=53)$ & $p^{*}$ \\
\hline \multicolumn{5}{|l|}{ Demographics and anthropometry } \\
\hline Age (years) & $40.1(1.9)$ & $41.7(2.3)$ & $39.3(1.4)$ & 0.34 \\
\hline $\operatorname{Sex}(M / F)$ & $49 / 33$ & $18 / 11$ & $31 / 22$ & 0.75 \\
\hline $\mathrm{BMI}\left(\mathrm{kg} \cdot \mathrm{m}^{-2}\right)$ & $30.9(0.5)$ & $32.7(1.1)$ & $29.9(0.5)$ & 0.02 \\
\hline Waist circumference $(\mathrm{cm})$ & $100.0(1.1)$ & $105.8(2.0)$ & $96.8(1.2)$ & $<0.001$ \\
\hline $\mathrm{SBP}(\mathrm{mmHg})$ & $127.4(1.5)$ & $132.1(2.7)$ & $124.8(1.8)$ & 0.02 \\
\hline DBP (mmHg) & $81.0(0.8)$ & $83.1(1.3)$ & $79.8(1.0)$ & 0.05 \\
\hline $\operatorname{SAT}\left(\mathrm{cm}^{3}\right)$ & $9268(405)$ & $10431(806)$ & $8632(427)$ & 0.55 \\
\hline $\operatorname{VAT}\left(\mathrm{cm}^{3}\right)$ & $3153(169)$ & $3847(301)$ & $2774(185)$ & 0.004 \\
\hline IHL (\%) & $5.3(0.5)$ & $10.3(0.9)$ & $2.6(0.2)$ & $<0.001$ \\
\hline \multicolumn{5}{|l|}{ Biochemistry } \\
\hline Glucose (mmol/L) & $4.3(0.1)$ & $4.6(0.1)$ & $4.1(0.1)$ & $<0.001$ \\
\hline Insulin (mU/L) & $8.4(0.5)$ & $10.6(1.2)$ & $7.2(0.5)$ & 0.01 \\
\hline $\operatorname{ALT}(\mathrm{U} / \mathrm{L})$ & $27.9(1.5)$ & $31.8(2.2)$ & $25.8(1.8)$ & 0.05 \\
\hline AST (U/L) & $24.7(1.1)$ & $25.9(1.8)$ & $24.0(1.3)$ & 0.40 \\
\hline hsCRP (mg/L) & $8.4(0.5)$ & $4.6(0.9)$ & $2.7(0.4)$ & 0.73 \\
\hline \multicolumn{5}{|l|}{ Lipids } \\
\hline Total cholesterol (mmol/L) & $5.5(0.1)$ & $5.5(0.2)$ & $5.5(0.1)$ & 0.99 \\
\hline Triglyceride $(\mathrm{mmol} / \mathrm{L})$ & $1.3(0.1)$ & $1.6(0.2)$ & $1.2(0.1)$ & 0.05 \\
\hline $\mathrm{LDL}(\mathrm{mmol} / \mathrm{L})$ & $3.5(0.1)$ & $3.5(0.2)$ & $3.5(0.1)$ & 0.99 \\
\hline $\mathrm{HDL}(\mathrm{mmol} / \mathrm{L})$ & $1.4(0.0)$ & $1.3(0.1)$ & $1.5(0.0)$ & 0.06 \\
\hline \multicolumn{5}{|l|}{ Habitual physical activity } \\
\hline Sedentary behavior (<1.6 METs) (hours:min) & $12: 08(0: 12)$ & $12: 10(0: 20)$ & $12: 08(0: 16)$ & 0.93 \\
\hline Light physical activity (1.6-2.9 METs) (hours:min) & $2: 58(0: 06)$ & $2: 48(0: 11)$ & 3:04 (0:07) & 0.20 \\
\hline Moderate physical activity (3-5.9 METs) (hours:min) & $1: 30(0: 07)$ & $1: 34(0: 15)$ & $1: 29(0: 06)$ & 0.75 \\
\hline Vigorous physical activity (6-8.9 METs) (hours:min) & $0: 05(0: 01)$ & 0:04 (0:01) & $0: 06(0: 0)$ & 0.39 \\
\hline Steps (per day) & $8915(355)$ & $8855(751)$ & $8947(371)$ & 0.90 \\
\hline Total energy expenditure $(\mathrm{kJ})$ & $13061(485)$ & $13145(541)$ & $13015(693)$ & 0.90 \\
\hline
\end{tabular}

M: male; F: female; BMI: body mass index; NAFLD: nonalcoholic fatty liver disease; SBP: systolic blood pressure; DBP: diastolic blood pressure; SAT: subcutaneous adipose tissue; VAT: visceral adipose tissue; IHL: intrahepatic lipid; ALT: alanine aminotransferase; AST: aspartate aminotransferase; hsCRP: high-sensitivity c-reactive protein; LDL: low density lipoprotein cholesterol; HDL: high density lipoprotein cholesterol; and METs: metabolic equivalents. Values are mean (standard error). ${ }^{*} p$ value for NAFLD versus non-NAFLD using Student's $t$-test for continuous variables and $\chi^{2}$ for categorical variables.

habitual activity levels are not related to VAT or liver fat; however it does not account for lifetime levels of habitual activity. Further, while objective assessment of activity via accelerometry is considered more accurate than self-report methods, participants may still have altered their usual behavior during monitoring. While a significant reduction in liver fat has been observed with just 4 weeks of structured aerobic exercise [32], it is uncertain how long current habitual activity patterns may take to affect VAT and liver fat.

On the basis of epidemiological data at the population level, physical inactivity is associated with the development of NAFLD [12-14] and visceral adiposity [11], and reducing sedentary behavior (including sitting time) should be generally advocated in all populations and is now recognized in public health messages [8]. However, our findings suggest that objectively measured physical activity is not greatly predictive of risk at an individual level and the associations between habitual physical activity levels, sedentary behavior, and metabolically detrimental fat depots such as VAT and
IHL remain uncertain. While our findings appear counterintuitive to epidemiological data, unlike these studies, we accurately quantified both liver fat and VAT, as well as 24hour habitual physical activity behavior, suggesting that the relationship may not be as straightforward.

There is now strong and consistent evidence that regular structured physical activity (exercise training) reduces VAT [2] and IHL [3-5]. This reflects current physical activity guidelines which advocate structured exercise for health benefits [1]. With the less potent stimulus of general habitual activity, the volume of activity required for benefit is much greater (e.g., the accumulation of $>470 \mathrm{~min}$ of physical activity for weight loss [25]). While increasing incidental activity is important for increasing total daily energy expenditure, there is an established dose potency of structured exercise for a range of cardiovascular and metabolic health risk variables [1]. Additionally, there are well-established inverse relationships between cardiorespiratory fitness and NAFLD [33], histological severity of liver disease [34], and 


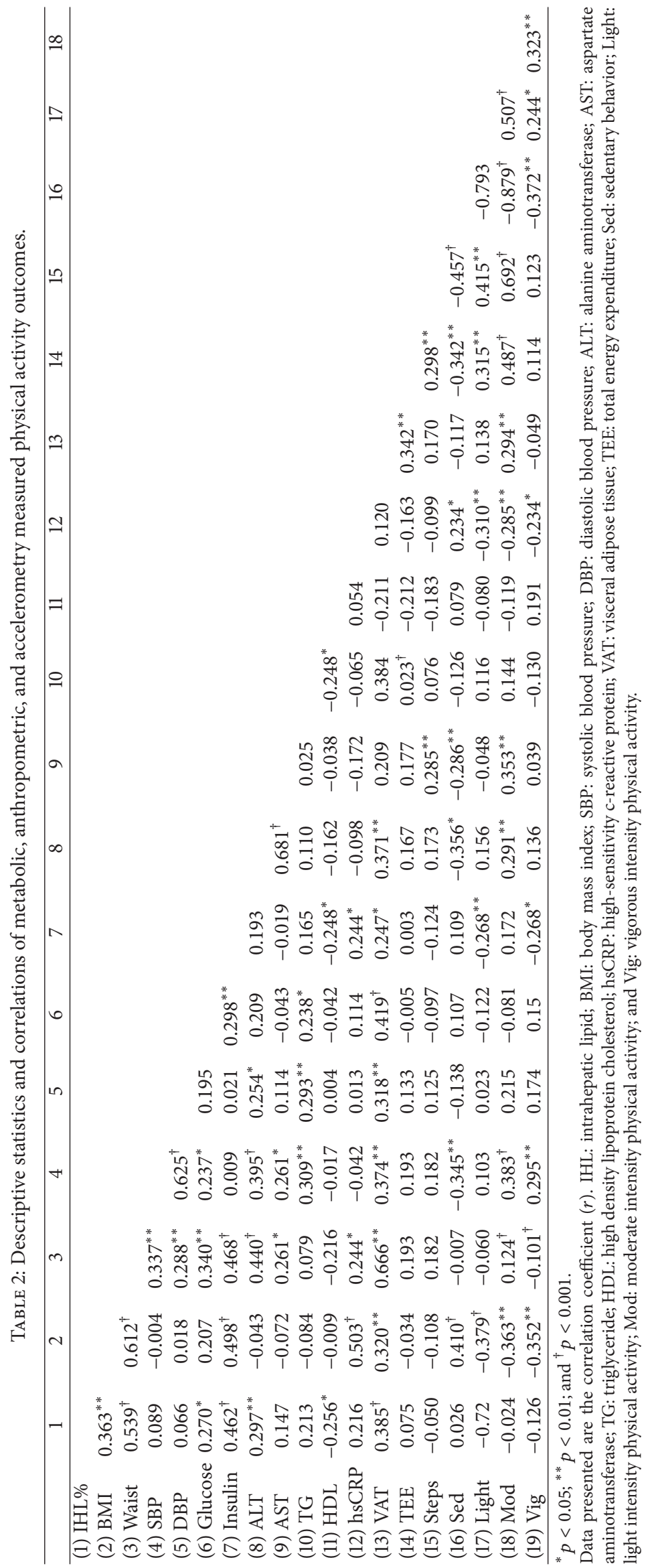


TABLE 3: Hierarchical regression model for prediction of visceral adiposity.

\begin{tabular}{|c|c|c|c|c|c|c|}
\hline & $R$ & $R^{2}$ & Adjusted $R^{2}$ & $R^{2}$ change & $p$ & $\beta$ \\
\hline Block 1 & 0.732 & 0.536 & 0.518 & 0.536 & $<0.001$ & \\
\hline Sex & & & & & & -0.251 \\
\hline Age & & & & & & 0.386 \\
\hline Waist & & & & & & 0.503 \\
\hline Block 2 & 0.790 & 0.624 & 0.571 & 0.087 & 0.032 & \\
\hline Sex & & & & & & -0.148 \\
\hline Age & & & & & & 0.244 \\
\hline Waist & & & & & & 0.525 \\
\hline TG & & & & & & 0.264 \\
\hline SBP & & & & & & 0.029 \\
\hline DBP & & & & & & -0.36 \\
\hline ALT & & & & & & 0.052 \\
\hline BMI & & & & & & 0.001 \\
\hline Glucose & & & & & & 0.127 \\
\hline Insulin & & & & & & -0.094 \\
\hline Block 3 & 0.808 & 0.653 & 0.587 & 0.029 & 0.136 & \\
\hline Sex & & & & & & -0.022 \\
\hline Age & & & & & & 0.194 \\
\hline Waist & & & & & & 0.510 \\
\hline TG & & & & & & 0.241 \\
\hline SBP & & & & & & -0.039 \\
\hline DBP & & & & & & -0.005 \\
\hline ALT & & & & & & 0.062 \\
\hline BMI & & & & & & 0.056 \\
\hline Glucose & & & & & & 0.174 \\
\hline Insulin & & & & & & -0.086 \\
\hline Sedentary & & & & & & -0.031 \\
\hline Moderate & & & & & & 0.212 \\
\hline Vigorous & & & & & & -0.023 \\
\hline
\end{tabular}

BMI: body mass index; SBP: systolic blood pressure; DBP: diastolic blood pressure; ALT: alanine aminotransferase; and TG: triglyceride. Boldface indicates statistical significance $(p<0.05)$.

VAT [35]. Indeed high levels of cardiorespiratory fitness is a characteristic of the phenomenon termed "metabolically healthy obesity" [36] and low cardiorespiratory fitness is an independent predictor of all-cause and cardiovascular disease mortality [37]. In consideration of this, the emphasis for behavior change in this population should arguably be on adopting and adhering to structured exercise programs, cognizant of individual barriers to participation in exercise and exercise preferences, which may require facilitation with behavioral management strategies.

Obesity in developed nations is linked with an obesogenic environment which encompasses a calorie-dense diet (overnutrition) and a physical activity hostile (or physical inactivity-promoting) environment. However, while both physical inactivity and excess adiposity are independently associated with a greater risk of mortality [38], it is contentious whether physical inactivity is a cause or an effect of obesity $[39,40]$. Therefore, while it is established that low amounts of physical activity and high amounts of sedentary behavior predict mortality [41], the role of these behaviors in the development of abdominal obesity and liver fat is unclear and perhaps minimal.

Recent research has suggested that prolonged sitting ( $\geq 8$ hours/day) is associated with an increased risk of allcause mortality [41, 42] and type 2 diabetes [42]. However, in support of our findings, the association between sedentary behavior and markers of cardiometabolic risk disappear when adjusted [43] or modified [42] for total physical activity levels. Hence only individuals with low activity and high levels of sedentary behavior such as sitting may be at increased health risk [44]. While there is a heightened understanding of the detrimental effects of increased sedentary behavior and sitting time $[42,45]$ and interrupting sitting time may confer some health benefit, this study suggests that a direct link between unstructured, objectively measured levels of physical activity and sedentariness and levels of metabolically risky fat depots is insignificant.

In concordance with these findings, despite significant associations with moderate-to-vigorous physical activity, no associations were observed between objectively quantified 
TABLE 4: Hierarchical regression model for prediction of liver fat.

\begin{tabular}{|c|c|c|c|c|c|c|}
\hline & $R$ & $R^{2}$ & Adjusted $R^{2}$ & $R^{2}$ change & $p$ & $\beta$ \\
\hline Block 1 & 0.550 & 0.303 & 0.276 & 0.303 & $<0.001$ & \\
\hline Sex & & & & & & 0.102 \\
\hline Age & & & & & & 0.026 \\
\hline Waist & & & & & & 0.575 \\
\hline Block 2 & 0.630 & 0.397 & 0.331 & 0.094 & 0.055 & \\
\hline Sex & & & & & & 0.176 \\
\hline Age & & & & & & 0.114 \\
\hline Waist & & & & & & 0.471 \\
\hline Glucose & & & & & & 0.23 \\
\hline HDL & & & & & & -0.162 \\
\hline ALT & & & & & & 0.104 \\
\hline BMI & & & & & & -0.146 \\
\hline Insulin & & & & & & 0.256 \\
\hline Block 3 & 0.632 & 0.400 & 0.305 & 0.003 & 0.957 & \\
\hline Sex & & & & & & 0.166 \\
\hline Age & & & & & & 0.136 \\
\hline Waist & & & & & & 0.481 \\
\hline Glucose & & & & & & 0.008 \\
\hline HDL & & & & & & -0.180 \\
\hline ALT & & & & & & 0.105 \\
\hline BMI & & & & & & -0.165 \\
\hline Insulin & & & & & & 0.260 \\
\hline Sedentary & & & & & & 0.059 \\
\hline Moderate & & & & & & 0.000 \\
\hline Vigorous & & & & & & 0.046 \\
\hline
\end{tabular}

sedentary time and hepatic steatosis (estimated via CT) in adults with NAFLD [23] or with VAT volume in men or women [46]. A large analysis in 3056 volunteers observed that individuals with NAFLD (defined by the fatty liver index [47]) spent less time in light, moderate, or vigorous activity than non-NAFLD counterparts and that the lowest quartile of physical activity was found in individuals with NAFLD and comorbid type 2 diabetes [22]. It has also been observed that individuals meeting 150 minutes/week of moderateto-vigorous physical activity as assessed via accelerometry had the lowest odds of hepatic steatosis (assessed via CT) independent of BMI (OR 0.77) [23]. However, in these investigations, it is unclear whether the low levels of physical activity caused the elevated liver fat content or whether NAFLD itself caused patients to have low participation in physical activity.

A limitation of this cross-sectional analysis is that the non-NAFLD cohort had low mean liver fat levels which reduced the range in the data. An important consideration is that we recruited adults with overweight/obesity who had no known comorbid cardiovascular or metabolic disease, which probably contributed to this limited spread. Further research examining the interaction between sedentary behavior, physical activity levels, and metabolically detrimental fat depots, employing gold-standard methods, across a more diverse population, is therefore warranted. Another limitation is that the aforementioned associations between physical activity, fitness, and NAFLD may not translate to disease severity. A retrospective analysis of 813 adults with biopsyproven NAFLD demonstrating that only those who reported meeting the minimal guidelines for vigorous intensity activity ( $\geq 75 \mathrm{~min} /$ week) and not moderate physical activity guidelines (150 to $\geq 300 \mathrm{~min} /$ week) reduced the odds of developing NASH (OR 0.65) with further reduced odds (OR $0.56)$ when the extensive guidelines for vigorous intensity activity ( $\geq 150 \mathrm{~min} /$ week) were met. Furthermore, the odds of advanced fibrosis were only reduced in patients meeting the extensive guidelines for vigorous activity [48]. These data suggest that vigorous activity per se may be necessary for improvements in the progressive forms of NAFLD although longitudinal data from clinical trials are lacking and limited by the invasive nature of liver biopsy to inform disease severity.

In conclusion, there were no associations between sedentary behavior and levels of IHL or VAT and low levels of objectively measured physical activity did not predict high levels of VAT or IHL. These findings suggest that while traditional risk factors such as waist circumference, triglycerides, fasting glucose, or fasting insulin may predict the incidence of NAFLD or high levels of VAT, objectively 
quantified physical activity levels and sedentary behavior did not predict those at risk.

\section{Data Access}

Shelley E. Keating and Nathan A. Johnson have access to all of the data and can vouch for the integrity of the data analyses.

\section{Disclosure}

The funding bodies had no role in the design of the study, collection and analysis of data, or decision to publish.

\section{Competing Interests}

The authors declare that there are no competing financial interests in relation to the work described. Shelley E. Keating, Helen M. Parker, Toby G. Pavey, Michael K. Baker, and Jacob George declare no conflict of interests. Nathan A. Johnson received honoraria for speaking engagements for Merck Sharp \& Dohme. Ian D. Caterson has performed and still performs clinical trials of obesity treatment and prevention, some funded by government, but others by the pharmaceutical industry. Current trials are funded by the NHMRC, Novo Nordisk, Pfizer, BMS, and SFI. He serves on the steering committees of international trials (SCOUT and EXSCEL). For the latter he received an honorarium. He has given talks for Novo Nordisk, Servier Laboratories, Ache, and Pfizer in the last 3 years. He serves on the Scientific Advisory Board of the Sansom Institute for Health Research and the Board of the Children's Medical Research Institute and chairs the Executive Management Committee of the bariatric surgical register for the Obesity Surgery Society of Australia and New Zealand.

\section{Authors' Contributions}

Shelley E. Keating, Jacob George, and Nathan A. Johnson carried out study concept and design; Shelley E. Keating and Helen M. Parker carried out acquisition of data; Shelley E. Keating and Nathan A. Johnson carried out study supervision; Shelley E. Keating, Toby G. Pavey, and Nathan A. Johnson carried out drafting of the manuscript; Ian D. Caterson and Jacob George carried out critical revision of the manuscript; Shelley E. Keating, Toby G. Pavey, Michael K. Baker, and Nathan A. Johnson carried out statistical analysis; Nathan A. Johnson and Jacob George obtained funding.

\section{Acknowledgments}

This research was supported by funding from the Diabetes Australia Research trust (Establishment Grant: Nathan A. Johnson) and Blackmores Australia Ltd. Jacob George is supported by the Robert W. Storr Bequest to the Sydney Medical Foundation, University of Sydney, a National Health and Medical Research Council of Australia (NHMRC) Program Grant (1053206) and a Project grant (1049857).

\section{References}

[1] C. E. Garber, B. Blissmer, M. R. Deschenes et al., "Quantity and quality of exercise for developing and maintaining cardiorespiratory, musculoskeletal, and neuromotor fitness in apparently healthy adults: guidance for prescribing exercise," Medicine and Science in Sports and Exercise, vol. 43, no. 7, pp. 1334-1359, 2011.

[2] I. Ismail, S. E. Keating, M. K. Baker, and N. A. Johnson, "A systematic review and meta-analysis of the effect of aerobic vs. resistance exercise training on visceral fat," Obesity Reviews, vol. 13, no. 1, pp. 68-91, 2012.

[3] S. E. Keating, D. A. Hackett, J. George, and N. A. Johnson, "Exercise and non-alcoholic fatty liver disease: a systematic review and meta-analysis," Journal of Hepatology, vol. 57, no. 1, pp. 157-166, 2012.

[4] S. Sullivan, E. P. Kirk, B. Mittendorfer, B. W. Patterson, and S. Klein, "Randomized trial of exercise effect on intrahepatic triglyceride content and lipid kinetics in nonalcoholic fatty liver disease," Hepatology, vol. 55, no. 6, pp. 1738-1745, 2012.

[5] S. E. Keating, D. A. Hackett, H. M. Parker et al., "Effect of aerobic exercise training dose on liver fat and visceral adiposity," Journal of Hepatology, vol. 63, no. 1, pp. 174-182, 2015.

[6] A. Kotronen, H. Yki-Järvinen, K. Sevastianova et al., "Comparison of the relative contributions of intra-abdominal and liver fat to components of the metabolic syndrome," Obesity, vol. 19, no. 1, pp. 23-28, 2011.

[7] I.-M. Lee, E. J. Shiroma, F. Lobelo et al., "Effect of physical inactivity on major non-communicable diseases worldwide: an analysis of burden of disease and life expectancy," The Lancet, vol. 380, no. 9838, pp. 219-229, 2012.

[8] Health AGDo, Australia's Physical Activity and Sedentary Behaviour Guidelines for Adults (18-64 years), Health AGDo, Canberra, Australia, 2014.

[9] L. S. Pescatello and D. Murphy, "Lower intensity physical activity is advantageous for fat distribution and blood glucose among viscerally obese older adults," Medicine \& Science in Sports \& Exercise, vol. 30, no. 9, pp. 1408-1413, 1998.

[10] F. F. Horber, S. A. Kohler, K. Lippuner, and P. Jaeger, "Effect of regular physical training on age-associated alteration of body composition in men," European Journal of Clinical Investigation, vol. 26, no. 4, pp. 279-285, 1996.

[11] I. A. Lesser, A. C. Yew, D. C. Mackey, and S. A. Lear, "A crosssectional analysis of the association between physical activity and visceral adipose tissue accumulation in a multiethnic cohort," Journal of Obesity, vol. 2012, Article ID 703941, 8 pages, 2012.

[12] G. Perseghin, G. Lattuada, F. De Cobelli et al., "Habitual physical activity is associated with intrahepatic fat content in humans," Diabetes Care, vol. 30, no. 3, pp. 683-688, 2007.

[13] S. D. Hsieh, H. Yoshinaga, T. Muto, and Y. Sakurai, "Regular physical activity and coronary risk factors in Japanese men," Circulation, vol. 97, no. 7, pp. 661-665, 1998.

[14] S. Zelber-Sagi, D. Nitzan-Kaluski, R. Goldsmith et al., "Role of leisure-time physical activity in nonalcoholic fatty liver disease: a population-based study," Hepatology, vol. 48, no. 6, pp. 1791$1798,2008$.

[15] C. Sreenivasa Baba, G. Alexander, B. Kalyani et al., "Effect of exercise and dietary modification on serum aminotransferase levels in patients with nonalcoholic steatohepatitis," Journal of Gastroenterology and Hepatology, vol. 21, no. 1, pp. 191-198, 2006. 
[16] A. St. George, A. Bauman, A. Johnston, G. Farrell, T. Chey, and J. George, "Independent effects of physical activity in patients with nonalcoholic fatty liver disease," Hepatology, vol. 50, no. 1, pp. 68-76, 2009.

[17] T. Leskinen, S. Sipilä, M. Alen et al., "Leisure-time physical activity and high-risk fat: a longitudinal population-based twin study," International Journal of Obesity, vol. 33, no. 11, pp. 12111218, 2009.

[18] K. Samaras, P. J. Kelly, M. N. Chiano, T. D. Spector, and L. V. Campbell, "Genetic and environmental influences on totalbody and central abdominal fat: the effect of physical activity in female twins," Annals of Internal Medicine, vol. 130, no. 11, pp. 873-882, 1999.

[19] S. M. Dyrstad, B. H. Hansen, I. M. Holme, and S. A. Anderssen, "Comparison of self-reported versus accelerometer-measured physical activity," Medicine \& Science in Sports \& Exercise, vol. 46, no. 1, pp. 99-106, 2014.

[20] M. A. Tully, J. Panter, and D. Ogilvie, "Individual characteristics associated with mismatches between self-reported and accelerometer-measured physical activity," PLOS ONE, vol. 9, no. 6, Article ID e99636, 2014.

[21] K. Hallsworth, C. Thoma, S. Moore et al., "Non-alcoholic fatty liver disease is associated with higher levels of objectively measured sedentary behaviour and lower levels of physical activity than matched healthy controls," Frontline Gastroenterology, vol. 6, no. 1, pp. 44-51, 2015.

[22] L. Gerber, M. Otgonsuren, A. Mishra et al., "Non-alcoholic fatty liver disease (NAFLD) is associated with low level of physical activity: a population-based study," Alimentary Pharmacology and Therapeutics, vol. 36, no. 8, pp. 772-781, 2012.

[23] M. T. Long, A. Pedley, J. M. Massaro et al., "Hepatic steatosis is associated with lower levels of physical activity measured via accelerometry," Obesity, vol. 23, no. 6, pp. 1259-1266, 2015.

[24] H. A. Smith, K. L. Storti, V. C. Arena et al., "Associations between accelerometer-derived physical activity and regional adiposity in young men and women," Obesity, vol. 21, no. 6, pp. 1299-1305, 2013.

[25] J. E. Donnelly, S. N. Blair, J. M. Jakicic, M. M. Manore, J. W. Rankin, and B. K. Smith, "Appropriate physical activity intervention strategies for weight loss and prevention of weight regain for adults," Medicine \& Science in Sports \& Exercise, vol. 41, no. 2, pp. 459-471, 2009.

[26] N. A. Johnson, D. W. Walton, T. Sachinwalla et al., "Noninvasive assessment of hepatic lipid composition: advancing understanding and management of fatty liver disorders," Hepatology, vol. 47, no. 5, pp. 1513-1523, 2008.

[27] V. Positano, A. Gastaldelli, A. M. Sironi, M. F. Santarelli, M. Lombardi, and L. Landini, "An accurate and robust method for unsupervised assessment of abdominal fat by MRI," Journal of Magnetic Resonance Imaging, vol. 20, no. 4, pp. 684-689, 2004.

[28] K. Norton, L. Norton, and D. Sadgrove, "Position statement on physical activity and exercise intensity terminology," Journal of Science and Medicine in Sport, vol. 13, no. 5, pp. 496-502, 2010.

[29] B. L. Wajchenberg, "Subcutaneous and visceral adipose tissue: their relation to the metabolic syndrome," Endocrine Reviews, vol. 21, no. 6, pp. 697-738, 2000.

[30] L. S. Szczepaniak, P. Nurenberg, D. Leonard et al., "Magnetic resonance spectroscopy to measure hepatic triglyceride content: prevalence of hepatic steatosis in the general population," American Journal of Physiology-Endocrinology and Metabolism, vol. 288, no. 2, pp. E462-E468, 2005.

[31] W. E. Consultation, "Waist circumference and waist-hip ratio," Report of a WHO Expert Consultation, World Health Organization, Geneva, Switzerland, 2008.

[32] N. A. Johnson, T. Sachinwalla, D. W. Walton et al., "Aerobic exercise training reduces hepatic and visceral lipids in obese individuals without weight loss," Hepatology, vol. 50, no. 4, pp. 1105-1112, 2009.

[33] K. Kantartzis, C. Thamer, A. Peter et al., "High cardiorespiratory fitness is an independent predictor of the reduction in liver fat during a lifestyle intervention in non-alcoholic fatty liver disease," Gut, vol. 58, no. 9, pp. 1281-1288, 2009.

[34] J. B. Krasnoff, P. L. Painter, J. P. Wallace, N. M. Bass, and R. B. Merriman, "Health-related fitness and physical activity in patients with nonalcoholic fatty liver disease," Hepatology, vol. 47, no. 4, pp. 1158-1165, 2008.

[35] S. Kim, J.-Y. Kim, D.-C. Lee, H.-S. Lee, J.-W. Lee, and J. Y. Jeon, "Distribution of abdominal obesity and fitness level in overweight and obese Korean adults," International Journal of Endocrinology, vol. 2014, Article ID 854392, 9 pages, 2014.

[36] F. B. Ortega, C. Cadenas-Sánchez, X. Sui, S. N. Blair, and C. J. Lavie, "Role of fitness in the metabolically healthy but obese phenotype: a review and update," Progress in Cardiovascular Diseases, vol. 58, no. 1, pp. 76-86, 2015.

[37] S. Kodama, K. Saito, S. Tanaka et al., "Cardiorespiratory fitness as a quantitative predictor of all-cause mortality and cardiovascular events in healthy men and women: a meta-analysis," The Journal of the American Medical Association, vol. 301, no. 19, pp. 2024-2035, 2009.

[38] P. T. Katzmarzyk, I. Janssen, and C. I. Ardern, "Physical inactivity, excess adiposity and premature mortality," Obesity Reviews, vol. 4, no. 4, pp. 257-290, 2003.

[39] L. H. Mortensen, I. C. Siegler, J. C. Barefoot, M. Grønbæk, and T. I. A. Sørensen, "Prospective associations between sedentary lifestyle and BMI in midlife," Obesity, vol. 14, no. 8, pp. 14621471, 2006.

[40] L. Petersen, P. Schnohr, and T. I. A. Sørensen, "Longitudinal study of the long-term relation between physical activity and obesity in adults," International Journal of Obesity, vol. 28, no. 1, pp. 105-112, 2004.

[41] H. P. van der Ploeg, T. Chey, R. J. Korda, E. Banks, and A. Bauman, "Sitting time and all-cause mortality risk in 222497 Australian adults," Archives of Internal Medicine, vol. 172, no. 6, pp. 494-500, 2012.

[42] A. Biswas, P. I. Oh, G. E. Faulkner et al., "Sedentary time and its association with risk for disease incidence, mortality, and hospitalization in adults: a systematic review and metaanalysis," Annals of Internal Medicine, vol. 162, no. 2, pp. 123-132, 2015.

[43] C. Maher, T. Olds, E. Mire, and P. T. Katzmarzyk, "Reconsidering the sedentary behaviour paradigm," PLoS ONE, vol. 9, no. 1, Article ID e86403, 2014.

[44] R. M. Pulsford, E. Stamatakis, A. R. Britton, E. J. Brunner, and M. Hillsdon, "Associations of sitting behaviours with allcause mortality over a 16-year follow-up: the Whitehall II study," International Journal of Epidemiology, vol. 44, no. 6, pp. 19091916, 2015. 
[45] S. Ryu, Y. Chang, H.-S. Jung et al., "Relationship of sitting time and physical activity with non-alcoholic fatty liver disease," Journal of Hepatology, vol. 63, no. 5, pp. 1229-1237, 2015.

[46] J. M. Murabito, A. Pedley, J. M. Massaro et al., "Moderate-tovigorous physical activity with accelerometry is associated with visceral adipose tissue in adults," Journal of the American Heart Association, vol. 4, no. 3, Article ID e001379, 2015.

[47] G. Bedogni, S. Bellentani, L. Miglioli et al., "The fatty liver index: a simple and accurate predictor of hepatic steatosis in the general population," BMC Gastroenterology, vol. 6, article 33, 2006.

[48] K. D. Kistler, E. M. Brunt, J. M. Clark, A. M. Diehl, J. F. Sallis, and J. B. Schwimmer, "Physical activity recommendations, exercise intensity, and histological severity of nonalcoholic fatty liver disease," American Journal of Gastroenterology, vol. 106, no. 3, pp. $460-468,2011$. 


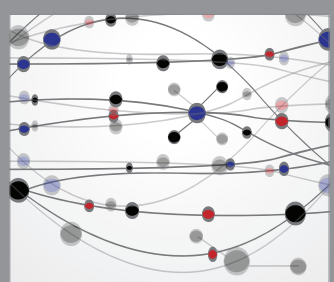

The Scientific World Journal
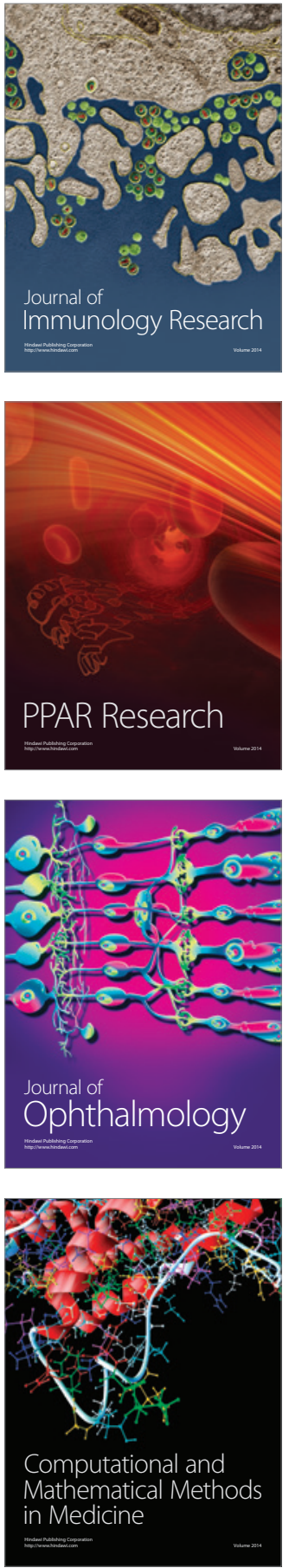

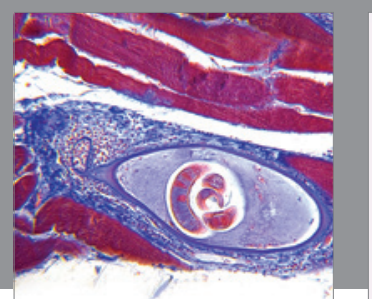

Gastroenterology Research and Practice

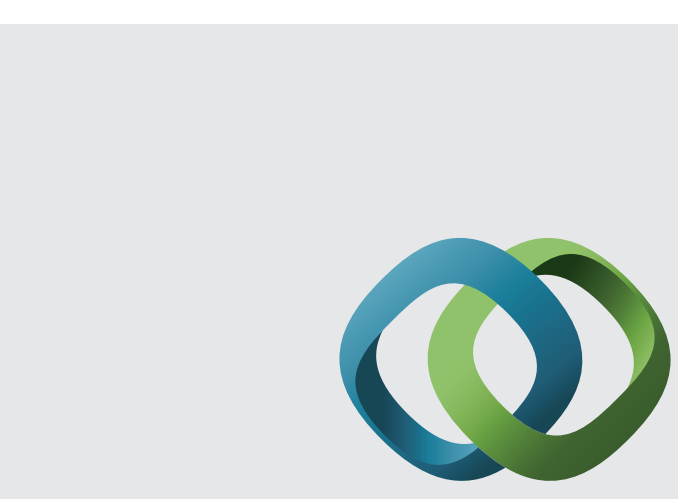

\section{Hindawi}

Submit your manuscripts at

http://www.hindawi.com
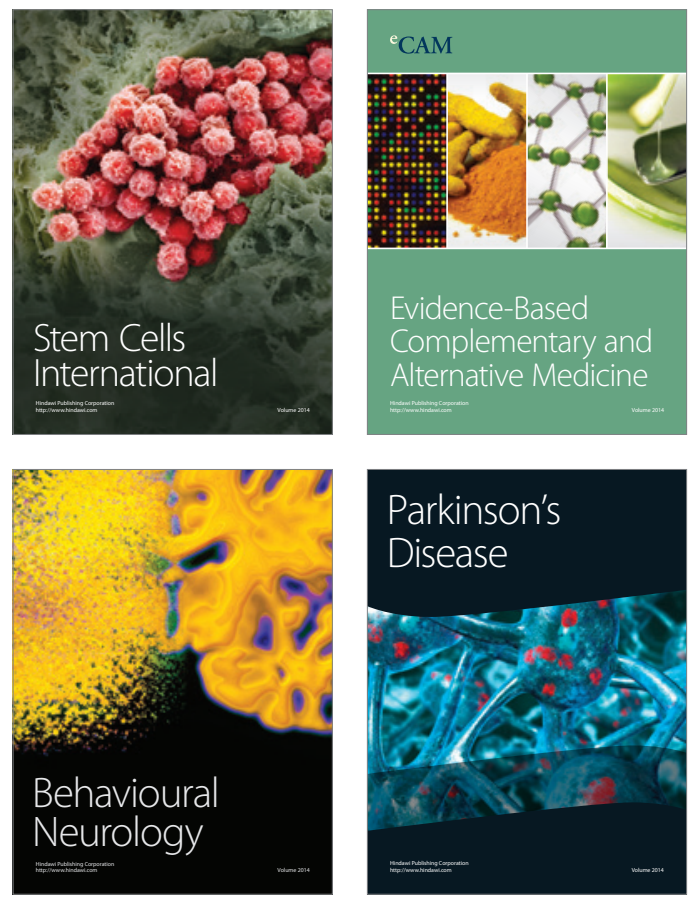
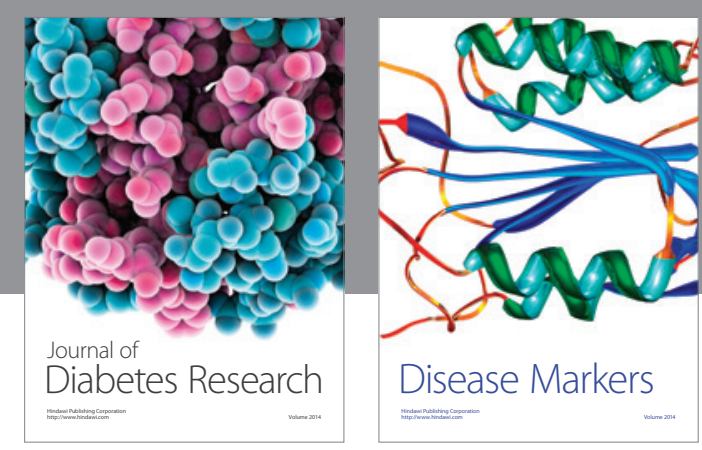

Disease Markers
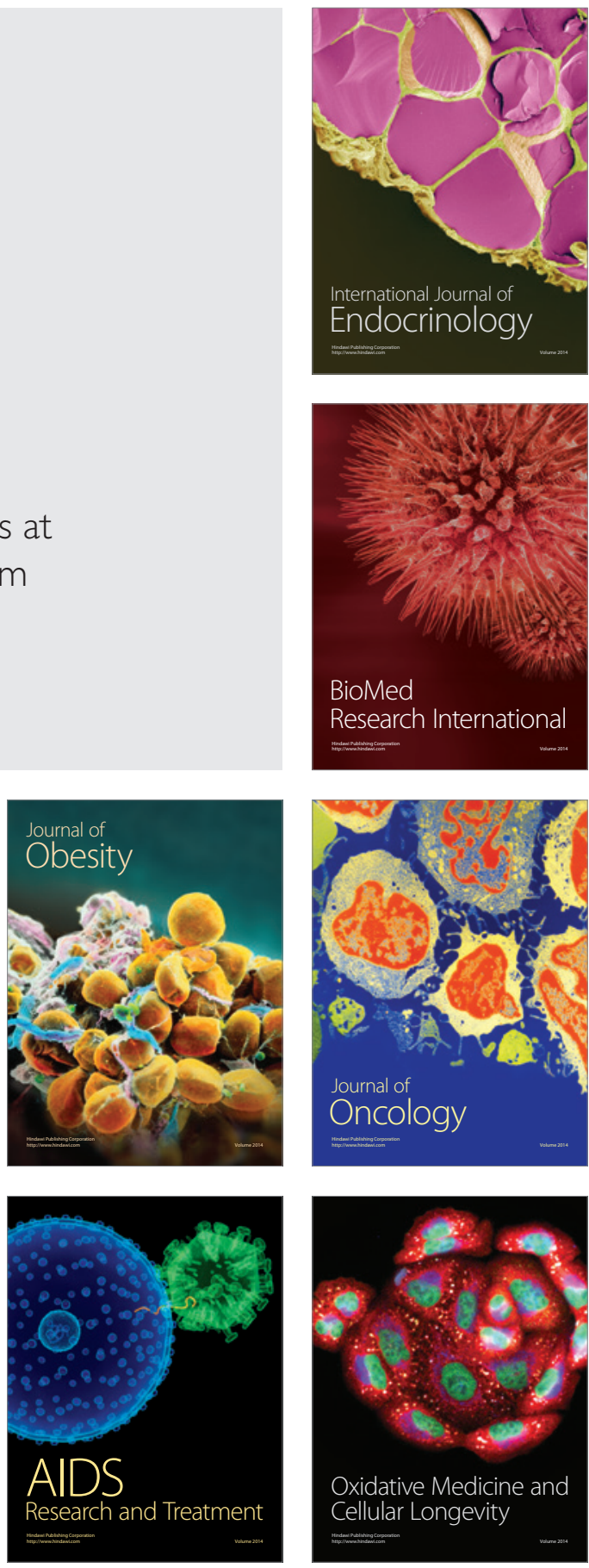